\title{
LIGHT AND ELECTRON MICROSCOPIC IMMUNOCYTOCHEMICAL LOCALIZATION OF GLUTAMIC ACID DECARBOXYLASE IN MONKEY GENICULATE COMPLEX: EVIDENCE FOR GABAERGIC NEURONS AND SYNAPSES ${ }^{1}$
}

\author{
ANITA E. HENDRICKSON, ${ }^{*}, 2$ MARILEE P. OGREN, ${ }^{*}$ JAMES E. VAUGHN, $\ddagger$ ROBERT P. BARBER, \\ AND JANG-YEN WU§ \\ * Department of Ophthalmology, University of Washington School of Medicine, Seattle, Washington 98195, $¥$ Division of \\ Neurosciences, City of Hope Research Institute, Duarte, California 91010, and \$ Department of Cell Biology, Baylor College \\ of Medicine, Houston, Texas 77030
}

Received October 11, 1982; Revised January 19, 1983; Accepted January 31, 1983

\begin{abstract}
The morphology and distribution of neuronal cell bodies and synapses that utilize $\gamma$-aminobutyric acid (GABA) as a transmitter have been studied in Macaca dorsal lateral geniculate nucleus (dLGN), ventral lateral geniculate nucleus (vLGN), and reticular nucleus of the thalamus (RNT). These cells are identified by immunocytochemical staining for the antiserum to the enzyme that synthesizes GABA, glutamic acid decarboxylase (GAD) and visualized by the peroxidase-antiperoxidase method for light and electron microscopy.

In the dLGN, GAD-positive (GAD+) cell bodies are found in all layers and in the interlaminar zones. The neuropil is more heavily labeled in the laminar than the interlaminar zones, with the magnocellular layers being most densely labeled. In the vLGN no stained cell bodies are seen, but the neuropil is intensely GAD+, especially in the retinal input layer. The RNT is just the opposite, with many GAD+ neurons but sparsely labeled neuropil.

The GAD+ neurons in dLGN have small cell bodies with a large infolded, unlabeled nucleus. GAD reactivity is found in the thin rim of cytoplasm and in the two to four thick, straight dendrites, and on the cytoplasmic surfaces of mitochondria, Golgi and membraneous cisternae, and vesicles.

GAD+ synaptic profiles are found in both pre- and postsynaptic relationships in the dLGN. Some GAD+ profiles are both pre- and postsynaptic in the same section, suggesting that these are presynaptic dendrites. In the laminae, GAD+ presynaptic profiles are very commonly found within synaptic glomeruli where they lie postsynaptic to a retinal axon terminal and presynaptic to an unlabeled dendrite, which also receives input from the retinal terminal. Outside of glomeruli, individual GAD+ profiles are presynaptic to either labeled or unlabeled cell bodies and dendrites. Other GAD+ profiles are postsynaptic to unlabeled F or RSD-type terminals. Individual GAD+ profiles ending on unlabeled or labeled dendrites are found in the interlaminar zones.

These data, when combined with previously published work on dLGN circuitry, suggest that GAD+ glomerular profiles are the presynaptic dendrites of the dLGN interneuron, whereas the individual GAD+ contacts arise from axons of RNT neurons.
\end{abstract}

The primate dorsal lateral geniculate nucleus (dLGN) has been the subject of numerous studies that have resulted in a substantial amount of information concerning its structural organization. It appears to have two main neuronal types: projection cells that send their axons to visual cortex, and intrinsic or interneurons

\footnotetext{
${ }^{1}$ This study was supported by National Institutes of Health Research Grants EY-01208, NS18858, EY-03909, NS13224, and in part by EY-01730, RR00166, and HD02274. A. E. H. is the recipient of the Dolly Green Scholar Award from Research to Prevent Blindness, Inc., and a travel grant from the Burroughs Wellcome Fund.

${ }^{2}$ To whom correspondence should be addressed.
}

whose processes remain within the dLGN (reviewed by Guillery, 1971; Wilson and Hendrickson, 1981). In monkey, more than $90 \%$ of the neurons are labeled after horseradish peroxidase injections into striate cortex, indicating that interneurons are present but scarce (Ogren and Hendrickson, 1976; Norden and Kaas, 1978). Several investigators have described a candidate for interneurons in Golgi preparations of monkey dLGN (Polyak, 1957; Campos-Ortega et al., 1968; Pasik et al., 1973; Saini and Garey, 1981; Wilson and Hendrickson, 1981). These cells are small with relatively few, straight, long primary dendrites that branch into elaborate processes with multiple 
dendritic appendages. These neurons have a locally ramifying beaded axon, or lack an identifiable axon. At the electron microscopic (EM) level, a corresponding cell type has been described that has a small soma, a large nucleus with an infolded nuclear membrane, and dendritic appendages that are both pre- and postsynaptic (LeVay, 1971; Pasik et al., 1973; Hamori et al., 1974). Although these authors are in general agreement that such cells are the same as those identified as interneurons in Golgi preparations, no combined EM-Golgi impregnation studies have been done to prove this claim directly. This interpretation is further substantiated, however, by studies which show that following removal of visual cortex, small neurons with presynaptic dendrites survive, whereas virtually all other neurons (i.e., striate cortex projection cells) degenerate, suggesting that these small cells do not have axons going to the destroyed cortex (Pasik et al., 1973; Dineen et al., 1982).

The synaptology of the dLGN also suggests the presence of interneurons. At the EM level, three main types of synapses are found. Two terminal types, called RLP and RSD, contain round vesicles and make asymmetric synaptic contacts (Guillery and Colonnier, 1970); their main sources are the axons of neurons in the retina and visual cortex, respectively (reviewed by Guillery, 1971; Wilson and Hendrickson, 1981). The third type, called F, contains pleomorphic or flat vesicles and makes symmetric contacts (Guillery and Colonnier, 1970). Other work has shown that there are at least two types of $\mathrm{F}$ profiles ${ }^{3}$ with slightly different morphology and different origins. Some $\mathrm{F}$ profiles arise from axons originating outside of the dLGN (Ohara et al., 1980; Montero and Scott, 1981), whereas others contain ribosomes and also receive synaptic contacts, indicating that they originate from the presynaptic dendrites of the dLGN interneurons (Ralston and Herman, 1969; Wong, 1970; LeVay, 1971; Famiglietli and Peters, 1972; Lieberman and Webster, 1972; Lieberman, 1973; Pasik et al., 1973; Hamori et al., 1974).

Evidence for a dLGN interneuron also can be found in electrophysiological recording data that demonstrate retinally driven inhibition onto the projection neuron which is generated by a cell that cannot be antidromically driven from the cortex (Stevens and Gerstein, 1976; Dubin and Cleland, 1977; reviewed by Burke and Cole, 1978). Iontophoretic analysis of this inhibition indicates that it is mediated by $\gamma$-aminobutyric acid (GABA) (Curtis and Tebecis, 1972). In cat dLGN, Sterling and Davis (1980) have shown that some small neurons specifically label with $\left[{ }^{3} \mathrm{H}\right] \mathrm{GABA}$ in vivo. These neurons are of the same size range as neurons which do not label after horseradish peroxidase injections into visual cortex (LeVay and Ferster, 1979), strengthening the likelihood that these cells are interneurons. In this same study, $\left[{ }^{3} \mathrm{H}\right]$ GABA also consistently labeled many $\mathrm{F}$ terminals, leading these authors to suggest that the population of GA$B A$ ergic intrinsic neurons gives rise to the $F$ terminal type of the dLGN. However, other data have indicated that not all inhibitory interactions are mediated by dLGN

\footnotetext{
${ }^{3}$ The term synaptic terminal will be reserved for synapses of undoubted axonal origin, and the term synaptic profile will be used for those which may be either axonal or dendritic in origin.
}

interneurons. In rat, some inhibitory neurons that affect projection cell activity clearly lie outside of the dLGN; one such population is the marked inhibitory pathway originating in the neurons of the reticular nucleus of the thalamus (RNT) (Sumitomo et al., 1976; Burke and Cole, 1978; Hale et al., 1982). In addition, Houser et al. (1980) have shown that most of the neurons in the RNT are positive for glutamic acid decarboxylase (GAD), the ratelimiting enzyme for GABA synthesis.

On the basis of these anatomical and electrophysiological data, it now seems possible that there is a small number of intrageniculate GABAergic neurons, but that there are also one or more extrageniculate sources for GABAergic inhibition. We have investigated this possibility by studying the dLGN, RNT, and other regions adjacent to dLGN in the Old World monkey Macaca, using light and EM immunocytochemistry based on a specific antiserum to GAD (Wu et al., 1973; Saito et al., 1974; Wu et al., 1982). Numerous investigations have verified this approach for localization of GABAergic neurons and synaptic terminals (reviewed by Ribak et al., 1981; Vaughn et al., 1981; Wu et al., 1982). We have found that monkey dLGN does contain a small population of GAD-positive (GAD+) somata and that the dLGN neuropil is richly supplied with GAD+ synaptic profiles. These profiles are all of the pleomorphic-flat type and many are synaptically related to, and show the same distribution as, the retinal input. We also find evidence for GAD+ presynaptic dendrites, suggesting that some of the GABAergic inhibition within the dLGN arises from an intrinsic population of interneurons. The RNT is the only adjacent nucleus containing GAD+ neurons, making it a likely second source of GABAergic input to dLGN. A preliminary report of this material has already appeared (Ogren et al., 1982). Similar findings have recently been described for GAD localization in rat dLGN (Ohara et al., 1983), and for $\left[{ }^{3} \mathrm{H}\right] \mathrm{GABA}$ in the cat (Sterling and Davis, 1980).

\section{Materials and Methods}

Six Macaca fascicularis monkeys were used for light (LM) and EM immunocytochemical localization of GAD, the rate-limiting synthesizing enzyme for GABA. Antiserum against purified GAD (Wu et al., 1973, 1982) was produced in rabbit and subjected to immunological testing for specificity (Saito et al., 1974; Wu et al., 1982). In addition, the specificity of the anti-GAD serum in immunocytochemical studies has been demonstrated previously in numerous regions of brains from several species, including monkey (McLaughlin et al., 1974; Barber and Saito, 1976; Houser et al., 1980; Hendrickson et al., 1981; Ribak et al., 1981; Wu et al., 1982).

One monkey received three injections ( $1 \mu \mathrm{l}$ each) of colchicine $(10 \mu \mathrm{g} / \mu \mathrm{l})$ into the region of the dLGN under sodium thiamylal (Surital, Parke-Davis) anesthesia $24 \mathrm{hr}$ before sacrifice in order to intensify GAD "staining" within somata and dendrites (Ribak et al., 1978). All animals were deeply anesthesized with barbiturate and were then perfused intracardially with $4 \%$ paraformaldehyde in $0.1 \mathrm{M}$ phosphate buffer $(\mathrm{pH} 7.4)$, containing $0.05 \%$ sodium periodate and $0.34 \%$ lysine which were added immediately before use. The brains were postfixed 
in the same fixative for $24 \mathrm{hr}$, and then blocks of thalamus were removed. Tissue for LM was infiltrated for 24 to 72 $\mathrm{hr}$ at $8^{\circ} \mathrm{C}$ in phosphate buffer containing $30 \%$ sucrose, and then frozen serial sections were cut coronally at 20 $\mu \mathrm{m}$ on a sliding microtome. Tissue for EM was stored in $10 \%$ sucrose-phosphate buffer at $8^{\circ} \mathrm{C}$ for 24 to $72 \mathrm{hr}$, and serial sections were cut coronally at $100 \mu \mathrm{m}$ on a Vibratome (Oxford Instruments).

Tissue preparation for LM and EM followed standard techniques for the indirect peroxidase-antiperoxidase method (Sternberger, 1979; Hendrickson et al., 1981; Vaughn et al., 1981). Briefly, tissue slices were incubated in primary GAD antiserum diluted 1:50 for $\mathrm{EM}$ or 1:200 for LM in phosphate-buffered saline (PBS) containing $1 \%$ normal goat serum and $0.1 \%$ Triton X-100 detergent which was used for all antiserum dilutions. Sections were incubated at $4^{\circ} \mathrm{C}$ for 16 to $36 \mathrm{hr}$ for $\mathrm{LM}$ and 1 to $2 \mathrm{hr}$ at room temperature for EM. Following a 1- to $2-\mathrm{hr}$ rinse in PBS containing $0.02 \%$ Triton $\mathrm{X}-100$, the sections were incubated in goat anti-rabbit serum diluted $1: 10$ for 30 to $60 \mathrm{~min}$ at $37^{\circ} \mathrm{C}$, rinsed $1 \mathrm{hr}$, incubated in rabbit peroxidase-antiperoxidase diluted $1: 50$ for $1 \mathrm{hr}$ at $37^{\circ} \mathrm{C}$, and rinsed first in PBS and then in Tris buffer. Finally, the sections were reacted with $0.03 \% 3,3^{\prime}$-diaminobenzidine tetrahydrochloride (Sigma), and $0.0006 \% \mathrm{H}_{2} \mathrm{O}_{2}$ in Tris buffer, $\mathrm{pH} 7.2$, for 10 to $30 \mathrm{~min}$ and rinsed in PBS for 30 $\mathrm{min}$. Incubations which were done for longer times, in higher concentrations of Triton, or entirely at $37^{\circ} \mathrm{C}$ resulted in heavier labeling, but the quality of ultrastructural preservation deteriorated under these conditions.

For LM, reacted sections were mounted on glass slides, dehydrated, and coverslipped. Some sections were counterstained with cresyl violet before dehydration, but most were examined in the absence of counterstaining.
Sections for EM were treated for $30 \mathrm{~min}$ in $2 \% \mathrm{OsO}_{4}$, dehydrated, and embedded in Epon. Selected areas of interest, such as labeled neuronal somata, were identified in semithin $(1 \mu \mathrm{m})$ sections, and then thin sections were cut either en face or perpendicular to the original block face. The perpendicular plane was favored because the large immunocytochemical reagents rarely penetrated more than 15 to $20 \mu \mathrm{m}$ into the tissue slice, which greatly limited the number of labeled sections that were obtained by en face sectioning. Thin sections were stained with uranyl acetate, either en bloc or on the grid, and with lead citrate on the grid. Some thin sections also were examined without uranyl and lead staining as a control to verify that electron-dense terminals identified as GAD+ had been produced by a specific combination of immunocytochemical reaction, diaminobenzidine and osmium.

Control tissue slices were run in parallel with every series. These consisted of either substituting normal rabbit serum in place of the primary GAD antiserum or using no serum in place of the primary GAD antiserum. Control sections at both light microscopic and EM levels showed only a light background density that was homogeneously distributed throughout the tissue (Fig. $1 \mathrm{C}$ ).

\section{Light microscopic observations}

Dorsal lateral geniculate nucleus. GAD+ immunoreactivity is present throughout the dLGN in scattered cell bodies and within labeled fibers and punctate structures in the neuropil; many of the latter have been shown to be synaptic profiles in our EM observations (see below). Although a few labeled cell bodies can be identified in each section, almost all of the GAD immuno-
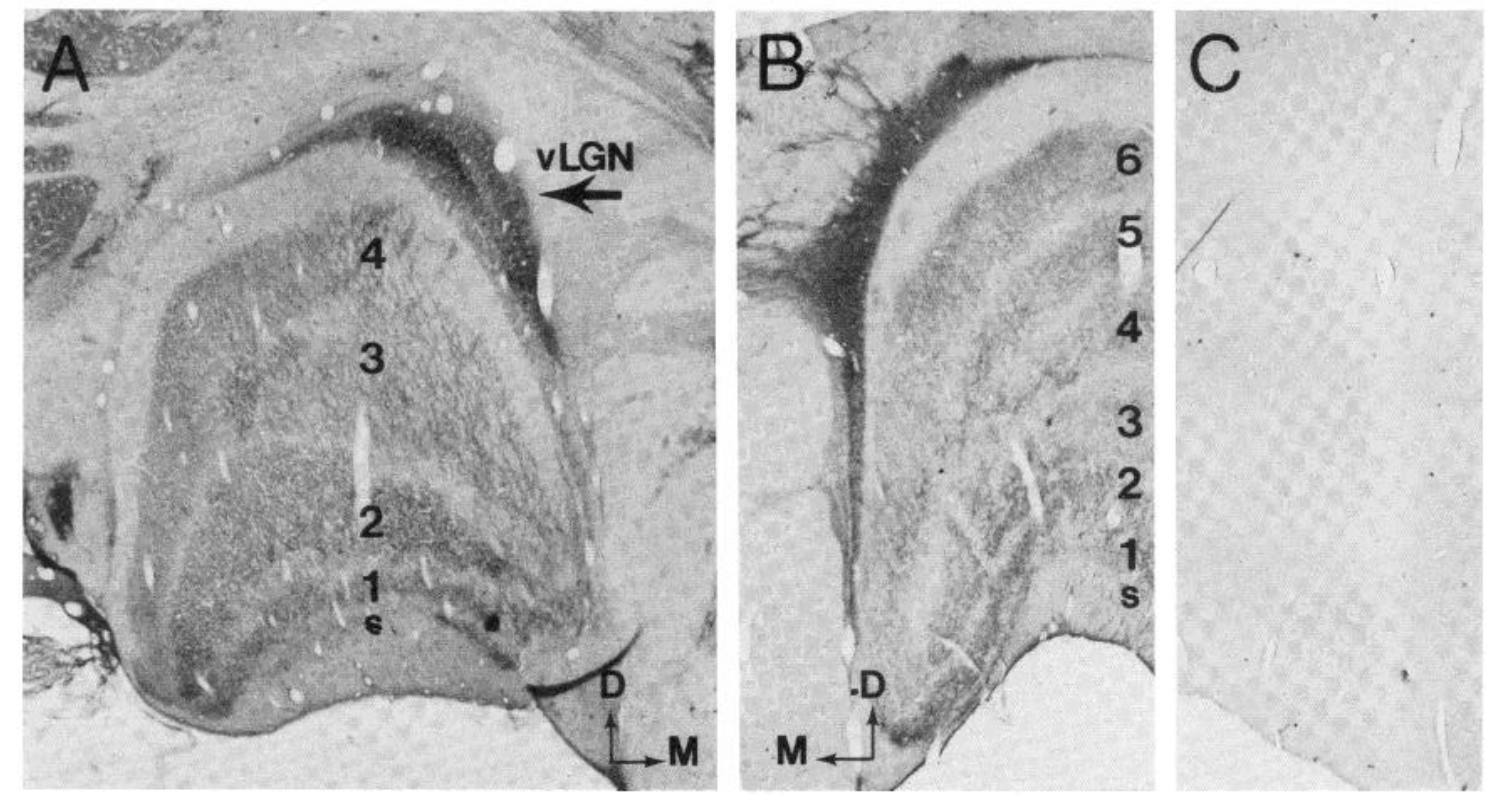

Figure 1. Low magnification light micrographs showing the distribution of GAD labeling in the dorsal (dLGN) and ventral $(v L G N)$ lateral geniculate nuclei $(A$ and $B)$. A control section incubated in normal rabbit serum exhibits only light background labeling $(C)$. These coronal sections through the anterior dLGN in $A$ and middle dLGN in $B$ show GAD labeling concentrated within the laminar zones. Magnocellular layers 1 and 2 and the S-layers $(S)$ appear more heavily labeled than parvocellular layers 3 to 6 . All of the interlaminar zones are relatively lightly labeled. In addition, the vLGN is heavily GAD+, especially the central portion. $M$, medial; $D$, dorsal. $\times 12$. 

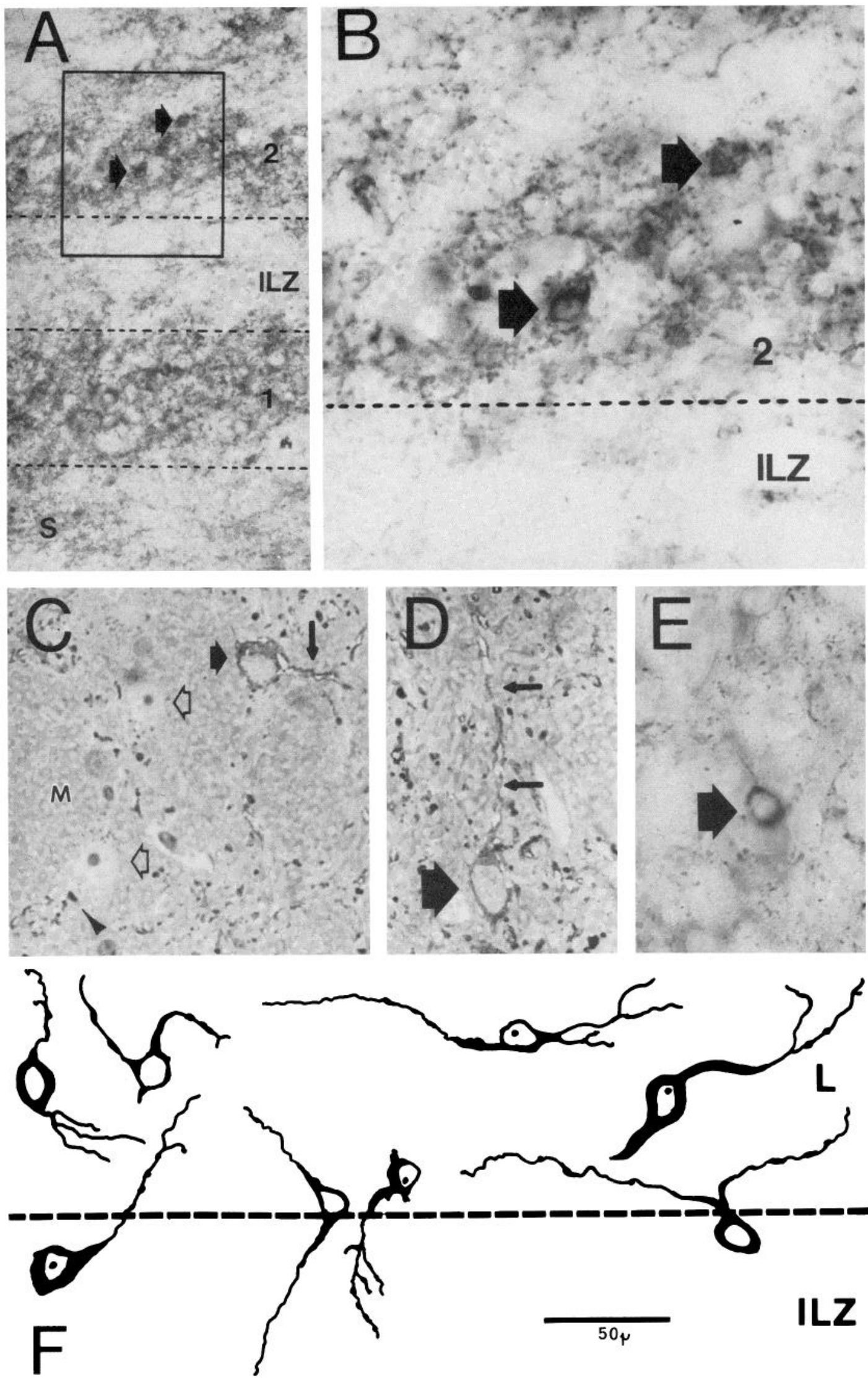

Figure 2. Higher magnification light micrographs of GAD labeling in the dLGN. $A$ and $B$, the neuropil labeling within the magnocellular layers 1 and 2 is more dense than that of the interlaminar zones (ILZ). The outlined zone in $A$ ( $\times 132)$ containing two small GAD-labeled cell bodies (arrows) is shown in more detail in $B(\times 240)$. These neurons are densely labeled with GAD reaction product within the cytoplasm but not the nucleus. The neuropil is filled with oval and round punctate GAD-labeled structures. $C$, This $1-\mu \mathrm{m}$ thick plastic section shows a small diameter labeled cell body (thick solid arrow) and its longitudinally 
reactivity is located within the neuropil (Figs. 1 and 2). Much of the GAD+ neuropil labeling lies within the geniculate layers, rather than the interlaminar zones, so that the characteristic geniculate layering is apparent even in the absence of Nissl counterstaining (Figs. 1, $A$ and $B$ and $2 A$ ). Neuropil labeling is found in magnocellular, parvocellular, intercalated and S laminae, but it is heaviest in the magnocellular layers. This distribution of GAD+ structures closely parallels the pattern of retinal input, which is restricted to the dLGN laminae (reviewed by Wilson and Hendrickson, 1981). It should be emphasized, however, that a sparse neuropil labeling for GAD is always present within all of the interlaminar zones (Fig. 2, $A$ and $B$ ).

A few GAD+ cell bodies are found within all layers, but the majority of cells remain unlabeled, even after colchicine pretreatment to intensify cell body staining (Ribak et al., 1978). In the magnocellular layers, the reactive neurons appear to be more densely labeled and may be more numerous than in the parvocellular layers, making them more easily detected (compare Fig. $2 B$ with $2 E$ ). Glial cells do not show GAD reactivity. Perikaryal labeling consists of a uniformly dense, brown reaction product within a thin rim of cytoplasm that surrounds a pale, unstained nucleus containing a single nucleolus. These GAD+ cells give off two to four thick, straight, proximal dendrites that branch at some distance from the cell body (Fig. 2, $C$ to $F$ ) resembling type C cells of Wilson and Hendrickson (1981) or type D1 neurons of Saini and Garey (1981). GAD+ dendrites sometimes cross laminar boundaries into interlaminar zones (Fig. $2 F$ ). Within the interlaminar zones and $\mathrm{S}$ layers labeled cell bodies are less frequently encountered but do occur (Fig. $2 F$ ).

GAD reactivity in the neuropil appears to be heavier in the magnocellular layers than parvocellular layers (compare Fig. $2 B$ with $2 E$ ). It is not clear whether this is due to the presence of more labeled fibers, a greater density of GAD labeling, or larger diameter fibers and terminals. At high magnification, thin, lightly labeled fibers are sometimes observed connecting rows and arcs of labeled puncta, forming beaded fibers, but more often the intervening spaces are unlabeled. Neuropil labeling within the interlaminar zones is of the same general morphology as that within the laminar zones, although it is more sparse. Occasionally GAD+ puncta are found directly contacting the surfaces of cell bodies, which may be either unlabeled or GAD+ (Fig. $2 C$ ).

Ventral lateral geniculate nucleus and reticular nucleus of the thalamus. The pregeniculate, or vLGN, appears to contain no labeled neuronal somata but is rich in a dense and intensely reactive GAD+ neuropil (Figs. $1 A$ and 3 ). The vLGN is clearly divided into two zones by GAD immunostaining; an outer zone is less densely
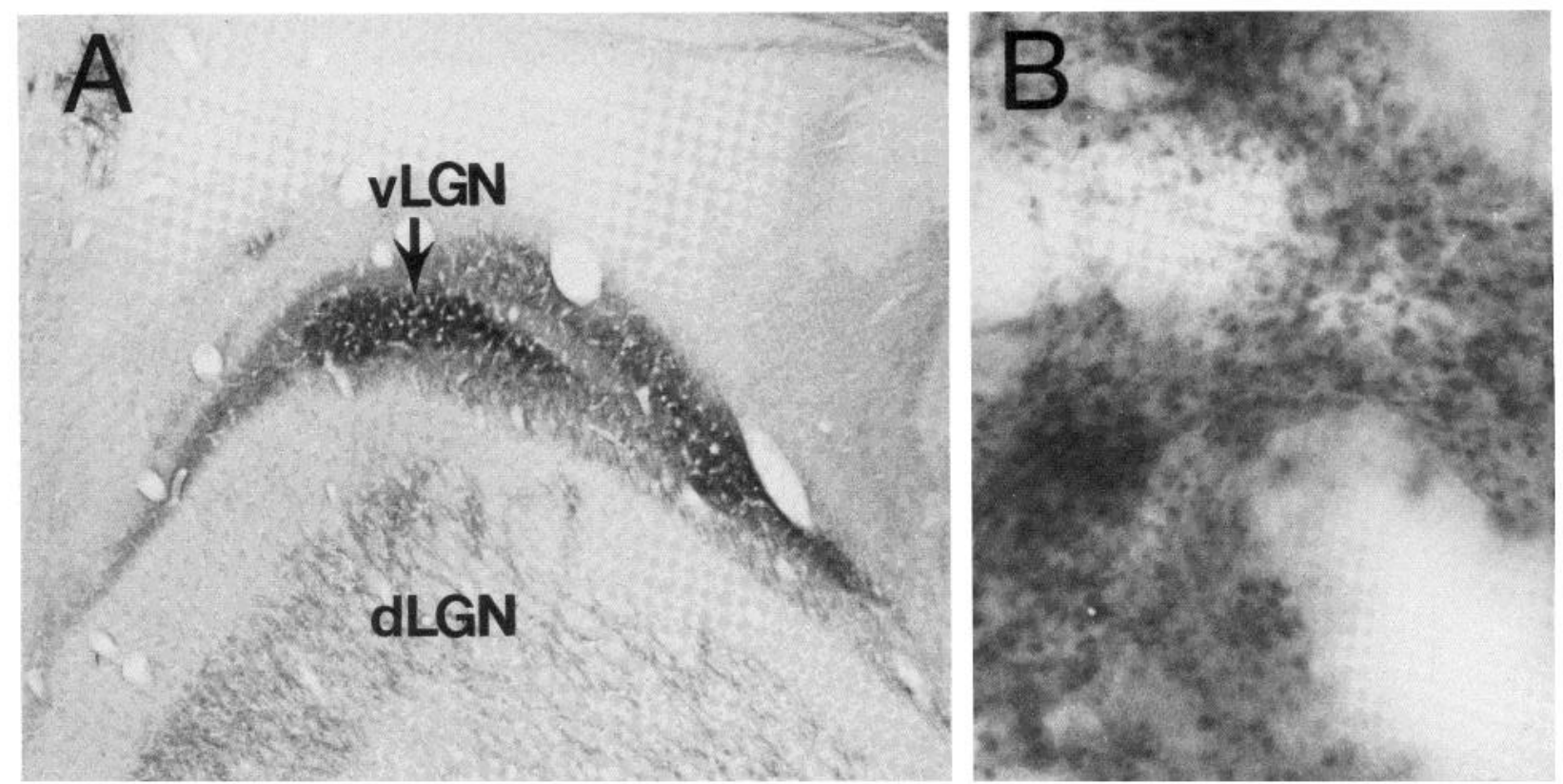

Figure 3. GAD labeling in the ventral lateral geniculate ( $L L G N)$. A, Dense GAD labeling is localized within the central zone, with lighter labeling in the surrounding layers of the vLGN. The layer of densest label (arrow) overlaps the zone of retinal input. Note that even the lightest labeling in the vLGN is equal to or heavier than that in the adjacent dorsal lateral geniculate $(d L G N$; also see Fig. $1 A)(\times 32)$. $B$, This higher magnification photomicrograph shows the dense GAD reactivity in the neuropil that is suggestive of large synaptic profiles. No labeled cell bodies have been found in the vLGN $(\times 1,680)$.

sectioned proximal dendrite (thin arrow). Two neighboring unlabeled cell bodies (open arrows) are also shown, one of which may be contacted at its surface by a labeled synaptic profile (arrowhead). Other labeled puncta, presumably synaptic profiles, are scattered throughout the neuropil but are absent from regions of myelinated fiber bundles $(M)(\times 260)$. $D$, This $1-\mu$ m thick plastic section shows another example of a labeled small neuron (thick arrow) and a longitudinally sectioned dendrite (thin arrows). Note densely stained GAD+ puncta in the surrounding neuropil $(\times 260)$. E, A labeled parvocellular neuron $($ thick arrow) and labeled neuropil elements from a frozen section are shown for comparison with the magnocellular labeling in $B(\times 240)$. $F$, Camera lucida drawings of GAD+ cells found throughout the dLGN. They have been grouped to show their relationships to laminar $(L)$ or interlaminar zones $(I L Z)$. 
labeled, whereas an inner zone that overlaps the retinal input (Hendrickson et al., 1970) is heavily labeled. The central portion of this inner zone contains especially dense label, resembling large synaptic terminals (Fig. $3 B$ ), but the results of an ongoing EM analysis will be required to precisely identify the cellular location of this label. Overall, the vLGN neuropil is much more GAD+ than the dLGN neuropil (see Figs. $1 A$ and $3 A$ ).

The RNT is also immunoreactive for GAD, but, in contrast to the vLGN and dLGN, the labeling of cell bodies in this nucleus overshadows that of the neuropil (Fig. 4, $A$ and $B$ ). Colchicine pretreatment does not significantly increase the number of stained cells but does show the cell bodies and especially their dendrites more clearly (Fig. 4, $C$ and $D$ ). In cresyl violet-counterstained sections, virtually every neuron in the RNT appears to be GAD+. The GAD+ neuropil in the RNT is rather sparse but is similar in morphology to that observed in the dLGN, consisting of oval and round puncta and a few beaded fibers. Both the GAD+ cell bodies and neuropil respect the narrow margins of the RNT and correspond to its classical distribution in cresyl violet-stained sections. In addition, a few scattered GAD+ neurons are seen lying between the dLGN and RNT within the internal capsule.

\section{Electron microscopic analysis of $d L G N$}

In the EM, neuronal cell bodies, cell processes, and synaptic profiles are all labeled for GAD. These struc- tures are considered as GAD+ when they contain accumulations of specific electron-dense material that is both diffusely distributed within the cytoplasm and associated with the membranes of certain organelles. The specificity of this staining is substantiated by its absence in control tissue incubated in nonimmune serum, and by the fact that in thin sections from material reacted in GAD antiserum but not stained with uranyl or lead salts, electron-dense material has the same distribution as seen in stained sections.

Cell bodies. The labeled cell bodies observed in the EM are generally those of small neurons in which most of the perikaryal volume is occupied by an unstained nucleus (Figs. 5, $A$ to $C$ and $10 B$ ). Infoldings of the nuclear membrane are common (Fig. $5, B$ and $C$ ). In tissue incubated for long times in antiserum diluted with buffer containing high concentrations of Triton, the thin rim of cytoplasm is densely packed with electron-dense reaction product (Figs. $5, A$ and $B, 10 B$ ). Because of the heavy label and the suboptimal ultrastructural preservation in such material, it is difficult to determine precisely what organelles are associated with the label. In tissue incubated for shorter times in less Triton, convincingly labeled somata are less numerous and the labeling that is present is light (Fig. $5 C$ ). This light labeling does permit identification of organelles that are associated with GAD+ product; these include the cytoplasmic membrane surfaces of the cisternae of the Golgi complex and of the mitochondria, vacuoles, and endoplasmic reticu-
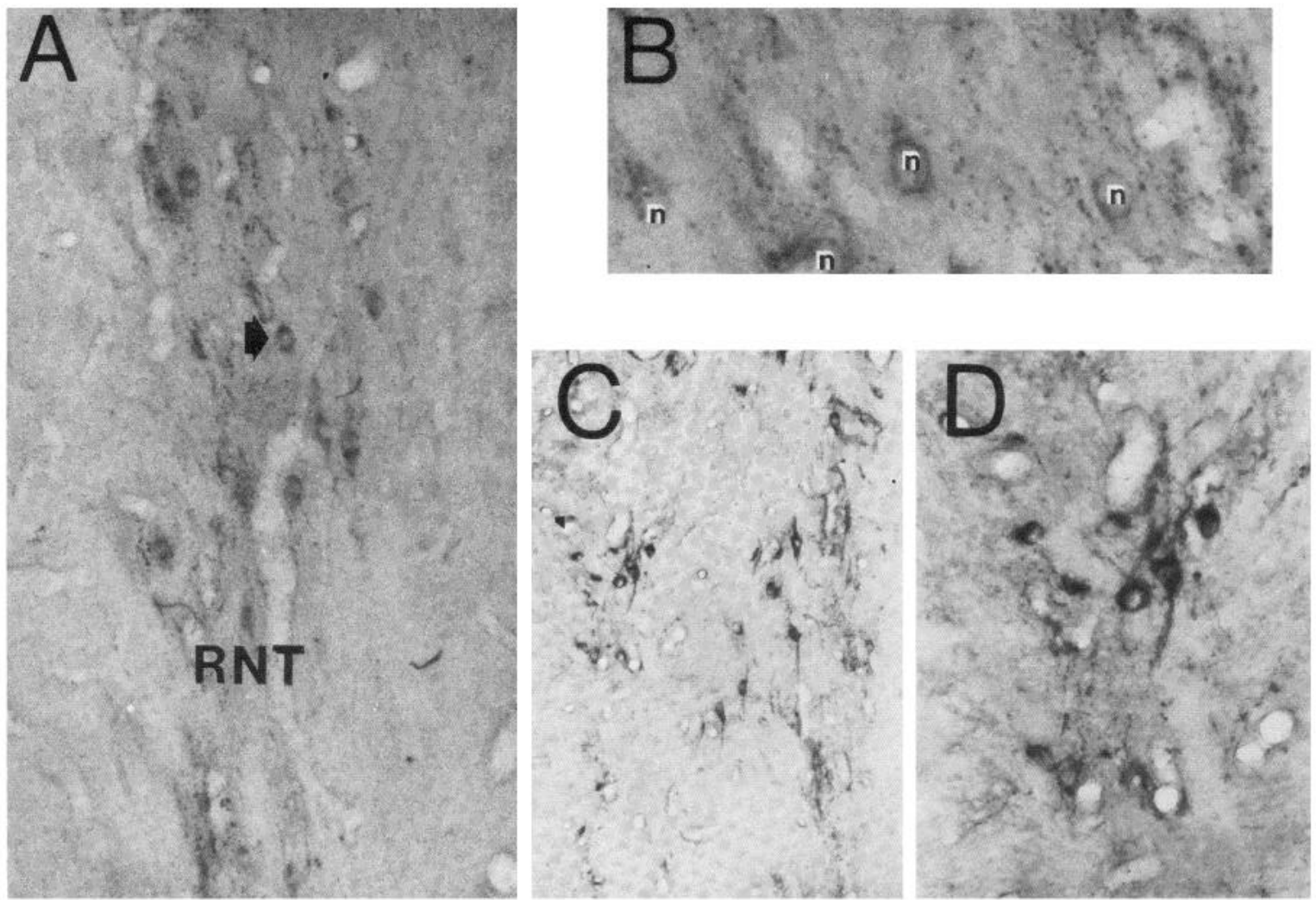

Figure 4. GAD labeling in the reticular nucleus of the thalamus $(R N T) . A, \mathrm{~A}$ low magnification coronal section through the RNT shows labeled cell bodies (arrow) and punctate labeling in the neuropil, both of which respect the narrow margins of the nucleus $(\times 200)$. B, A higher magnification photograph of RNT shows that every detectable neuron ( $n$ ) has GAD-labeled cytoplasm surrounding an unlabeled nucleus. The neuropil contains sparse punctate labeling $(\times 450)$. $C$ and $D$, Sections from an animal with colchicine pretreatment show that RNT somata and dendritic labeling is greatly intensified, but neuropil labeling appears to be diminished $(C, \times 125 ; D, \times 200)$. 

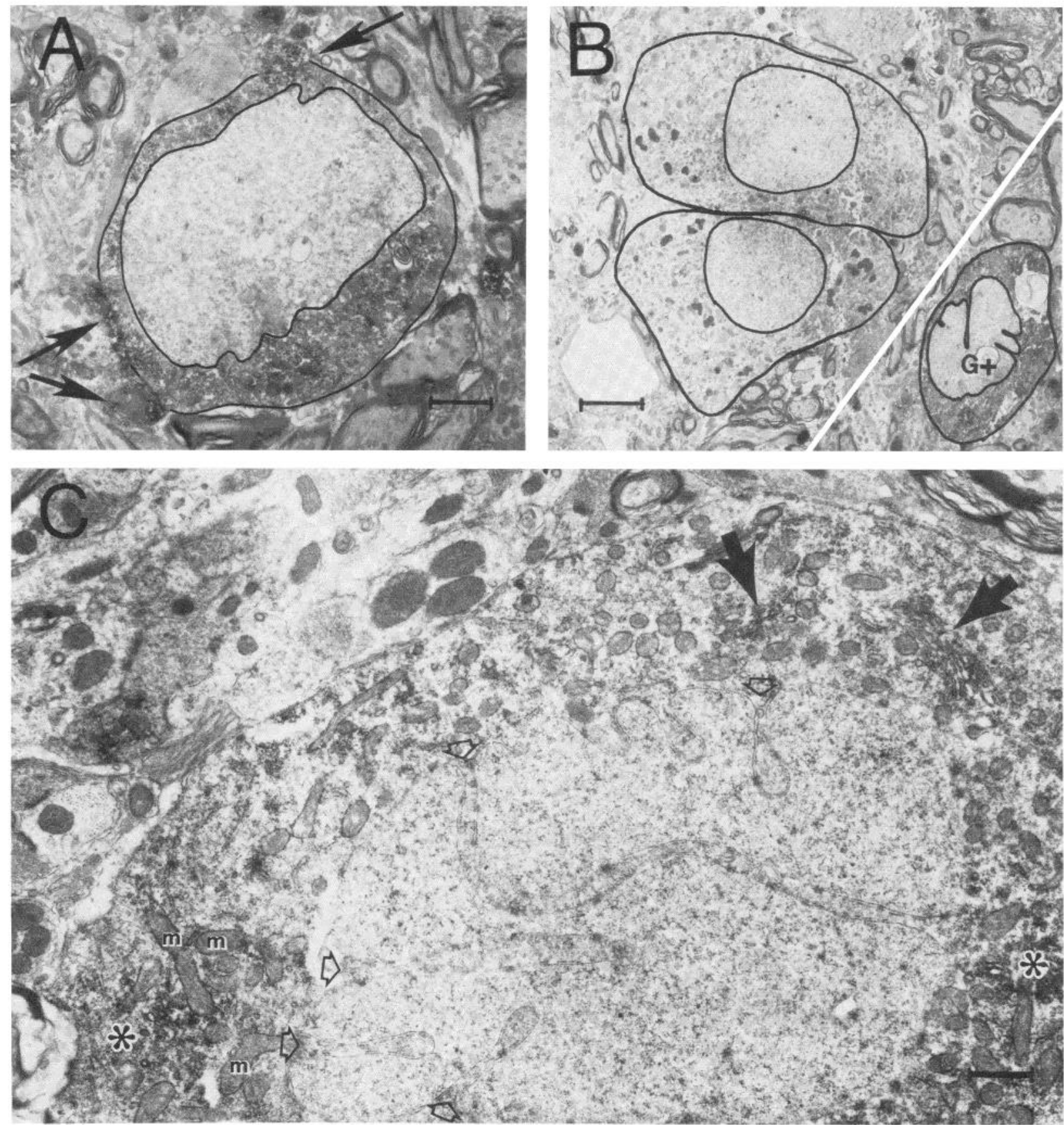

Figure 5. Low magnification electron micrographs of GAD-positive neurons in the dLGN. $A$, This labeled cell body contains dense accumulations of GAD + material in the thin rim of cytoplasm that surrounds the unstained nucleus. Three GAD-labeled profiles in the immediately adjacent neuropil contact the surface of this cell (arrows). The cytoplasmic and nuclear borders have been outlined for clarity. Scale bar $=5 \mu \mathrm{m}$. B, This composite electron micrograph shows a GAD+ neuron $(G+)$ and two unlabeled neighboring dLGN neurons, allowing a direct comparison of labeled versus unlabeled cytoplasm, relative cell size, and nuclear shape. The cell and nuclear membranes have been outlined for clarity. Scale bar $=5 \mu$ m. $C$, Higher magnification electron micrograph of GAD + neuronal cytoplasm. Incubation in lower levels of detergent has resulted in lighter GAD labeling but better ultrastructural preservation than shown in $A$ and $B$. GAD+ reactivity is seen on the outer surfaces of mitochondria $(m)$, cisternae of the Golgi complex (solid arrows), and in scattered ill-defined regions of the cytoplasm (asterisks). Note deep infoldings in the nuclear membrane (open arrows). Scale bars $=1.0 \mu \mathrm{m}$.

lum. The nuclear membrane, the nucleoplasm, and mitochondrial cisternae remain unlabeled (Fig. 5C).

Cell processes. Conventional dendrites that contain ribosomes and/or rough endoplasmic reticulum, receive synapses, and are also GAD+ are not commonly seen, but we have found both large and small diameter examples of this type of dendrite (Fig. 6A). Reaction product is found on the cytoplasmic membrane surfaces of the mitochondria, smooth endoplasmic reticulum cisternae, and vesicular structures. Fibrous material that may be poorly preserved microtubules also is associated with reaction product. Longitudinal sections through varicose processes that may either be axons or dendrites (Fig.6B) show GAD labeling in regions where organelles are nu- 

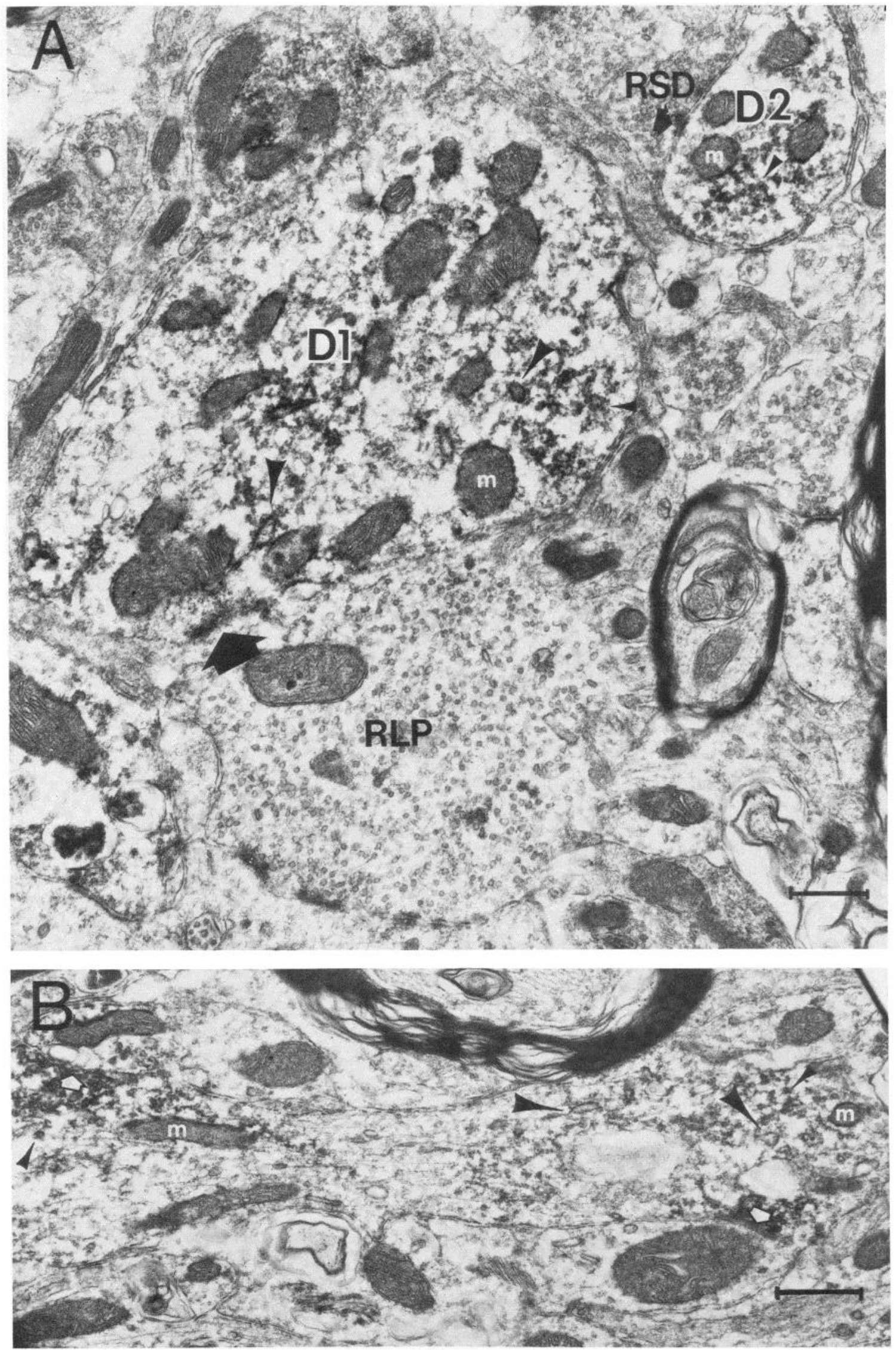

Figure 6 
mcrous, whereas the labeling diminishes or disappears in intervening regions where organelles are scarce. If a thin section passed transversely through an unlabeled region, the fiber would appear unlabeled, suggesting that these regions may correspond to the unlabeled or lightly labeled zones intervening between the GAD+ puncta observed in light micrographs.

In addition to GAD+ conventional dendrites, other GAD+ profiles both receive and make synaptic contacts (Figs. 7 to 9). We regard these as presynaptic dendrites because of this characteristic pre- and postsynaptic relationship and because these $\mathrm{GAD}+$ profiles exhibit other morphological characteristics that correspond to the presynaptic dendrites described in many conventional EM studies of thalamus (Ralston and IIerman, 1969; Wong, 1970; LeVay, 1971; Famiglietti and Peters, 1972; Lieberman and Webster, 1972; Lieberman, 1973; Pasik et al., 1973; Hamori et al., 1974; Partlow et al., 1977; Ogren and Hendrickson, 1979; Winfield, 1980; Wilson and Hendrickson, 1981). This identification must be a provisional one, however, because we do not have direct morphological evidence for the continuity between these profiles and a GAD+ cell body. Another commonly used marker for presynaptic dendrites is the presence of ribosomes or rough-surfaced endoplasmic reticulum. The presynaptic $\mathrm{GAD}+$ profile in Figure $7 A$ does appear to contain roughsurfaced endoplasmic reticulum, but this marker is typically found in proximal dendrites which have few synapses (LeVay, 1971), reducing the likelihood of both the characteristic synaptology and organelles occurring in the same section. Ribosomes are sparse in distal dendrites and are difficult to distinguish from GAD+ reaction product, minimizing their usefulness as a criterion for dendritic identification in more peripheral dendrites where presynaptic contacts are more common. Therefore, we have relied on the distinctive synaptic relationships rather than the organelle content to identify GAD+ presynaptic dendrites in this study.

Synaptic profiles. GAD+ synaptic profiles form a variety of synaptic relationships (Figs. 7 to 12) and are widely distributed throughout the neuropil. They typically contain one to five dark mitochondria and scattered synaptic vesicles, although in some instances the vesicles may be quite densely packed. Because GAD+ reactivity is associated with the surfaces of synaptic vesicles and is often found in the vicinity of the synaptic specialization as well, the appearance of these two very important morphological characteristics is frequently altered or obscured. This results in a great deal of variability in the appearance of GAD+ synaptic profiles, and as such it is difficult to classify them according to strict, traditional criteria. For example, whereas some GAD+ synaptic vesicles are clearly flat (Fig. $8 D, G 1$ ), others are more rounded or pleomorphic (Fig. $7 C, G 1$ ). In addition, some profiles form clearly symmetric synaptic specializations (Fig. 7A), whereas others are apparently more asymmetric (Fig. $8 A$ ). Nonetheless, their overall morphology and synaptic relationships indicate that the GAD+ profiles fit the different subtypes of $F$ terminals described in conventional EM material (discussed by Lieberman and Webster, 1972; Ogren and Hendrickson, 1979; Sterling and Davis, 1980; Wilson and Hendrickson, 1981). Because it is not possible in a single thin section of this material to consistently determine the difference between axon terminals and isolated synaptic sites in presynaptic dendrites, we will refer to the whole population as GAD+ synaptic profiles.

GAD+ profiles form the presynaptic (Figs. $6 B, 7$ to 10 , and 12) as well as the postsynaptic (Figs. 7 to 9 and 11) elements of synaptic junctions and frequently are both pre- and postsynaptic in the same section (Figs. 7, 8A, and $9 A$ ). For reasons described above, we provisionally regard those that are both pre- and postsynaptic in the same section as presynaptic dendrites. In general, GAD+ synaptic profiles are postsynaptic to all other unlabeled terminal and profile types-that is, RLP, RSD, and F. Occasionally GAD+ profiles synapse onto other GAD+ synaptic profiles (Figs. 8 and $12 B$ ). In these cases, the postsynaptic GAD+ element may also be presynaptic (Fig. 8, $B$ and $D$ ), indicating that this intermediate element is a presynaptic dendrite. GAD+ terminals also are presynaptic to cell bodies (Fig. 10) and to conventional dendrites (Figs. 7 to 9 and 12) that may either be labeled or unlabeled. GAD+ synaptic profiles have not been observed in presynaptic apposition to unlabeled synaptic terminals of any type.

Throughout the neuropil, GAD+ synaptic profiles are most commonly found in synaptic complexes or glomeruli as the intermediate elements in synaptic triads or in serial synapses (Figs. 7, $B$ and $C$ and 9 ). In these configurations, they are postsynaptic to an unlabeled retinal axon terminal and presynaptic to an unlabeled large dendrite that is often postsynaptic to the retinal axon terminal. GAD+ profiles within synaptic complexes may synapse with one or more dendrites in the same section. A single GAD+ process can display several different synaptic relationships. For instance, in Figure 9, a lightly stained GAD+ longitudinal process that resembles a dendrite takes part in a synaptic triad with a retinal terminal and a proximal dendrite and is also postsynaptic

Figure 6. Electron micrograph of GAD + cell processes in the dLGN. $A$, This transverse section through two dendrites $(D 1$ and D2) shows GAD labeling on the outer surfaces of mitochondria $(m)$, small cisternae (large arrowheads), and vesicular structures (small arrowheads). Because of the dense label, ribosomes are difficult to identify with certainty, but they probably lie just above $D 1$. D1 is a proximal dendrite postsynaptic to an unlabeled retinal terminal $(R L P)$ at the large arrow. D2 is a distal dendrite that is postsynaptic to an unlabeled RSD terminal at the small arrow. $B$, A longitudinal section through a GAD+ varicose process shows GAD labeling associated with mitochondria $(\mathrm{m})$, small cisternae (large arrowheads), and vesicular structures $($ small arrowheads) that lie in the wider portions of the process. The narrower central portion contains neurofibrils but few organelles, and it is unlabeled. Accumulations of GAD reactivity associated with vesicles at the upper left and lower right of this process are suggestive of presynaptic sites (white arrows), indicating that this GAD+ profile could be either an axon or a varicose presynaptic dendrite. Scale bars $=0.5 / \mathrm{m}$. 

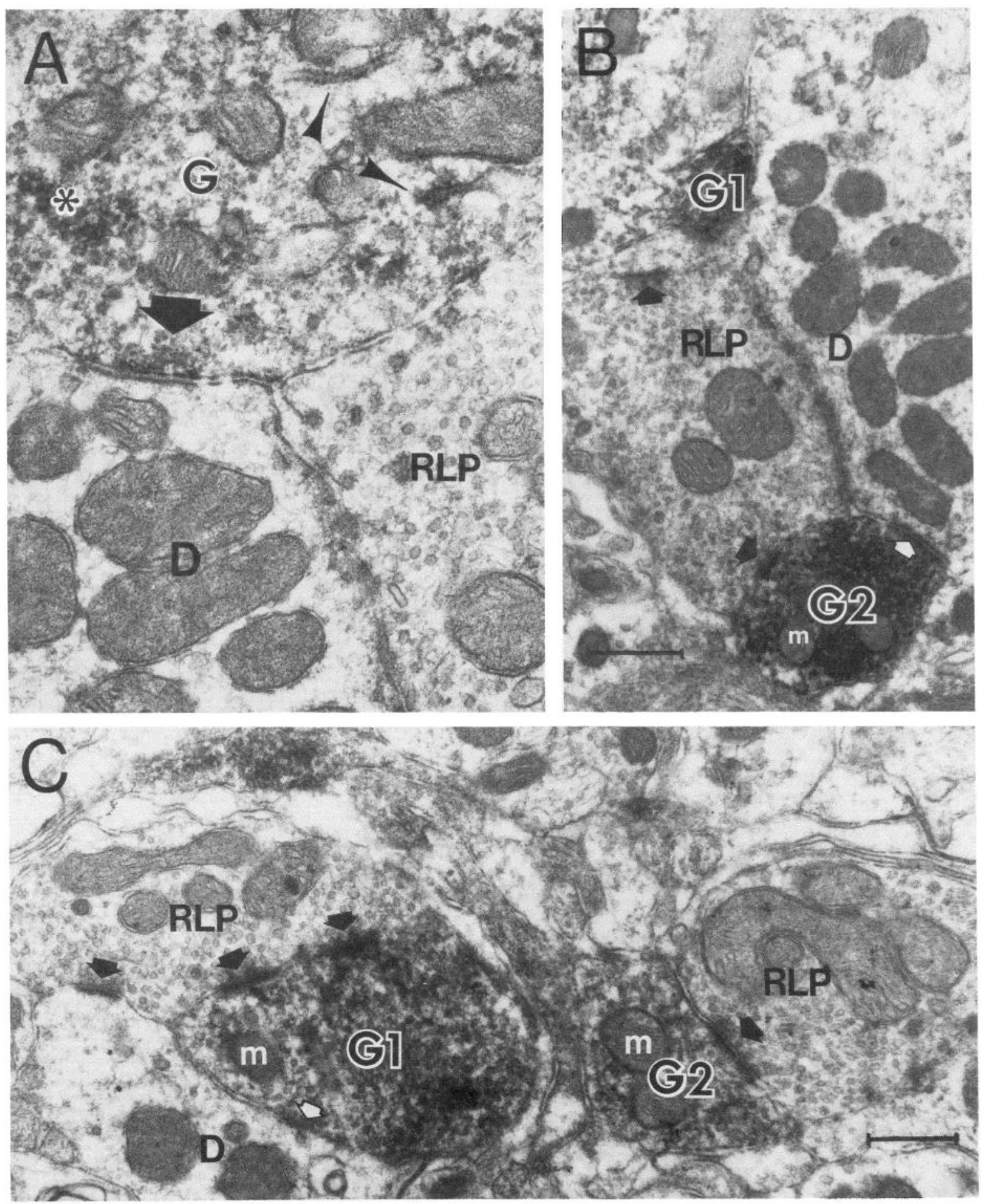

Figure 7. Electron micrographs of presynaptic GAD+ profiles forming synaptic complexes. $A$, This GAD+ $(G)$ synaptic profile is adjacent to a retinal synaptic terminal $(R L P)$ and is presynaptic to an unlabeled large dendrite $(D)$ at a clearly symmetric synaptic specialization (black arrow). Note also the filamentous adhesions between the dendrite and the RLP. Arrowheads mark structures that may be strands of rough-surfaced endoplasmic reticulum. GAD reactivity associated with synaptic vesicles in $\mathrm{G}$ is marked by an asterisk. $B$, Two GAD + (G1 and G2) profiles are so densely packed with reaction product that it obscures the synaptic vesicles, but G2 can be seen to form a synaptic contact at the white arrow with a large unlabeled proximal dendrite. An RLP terminal is presynaptic to G2 and to a dendritic spine from the same large proximal dendrite (black arrows), thus completing a synaptic triad. $C$, Two GAD+ profiles (G1 and G2) are postsynaptic to RLP terminals at asymmetric contacts (black arrows). The RLP terminal on the left is presynaptic to a dendrite $(D)$ that also receives a contact from G1 (white arrow), forming a synaptic triad. Scale bars $=0.5 \mu \mathrm{m}$. 

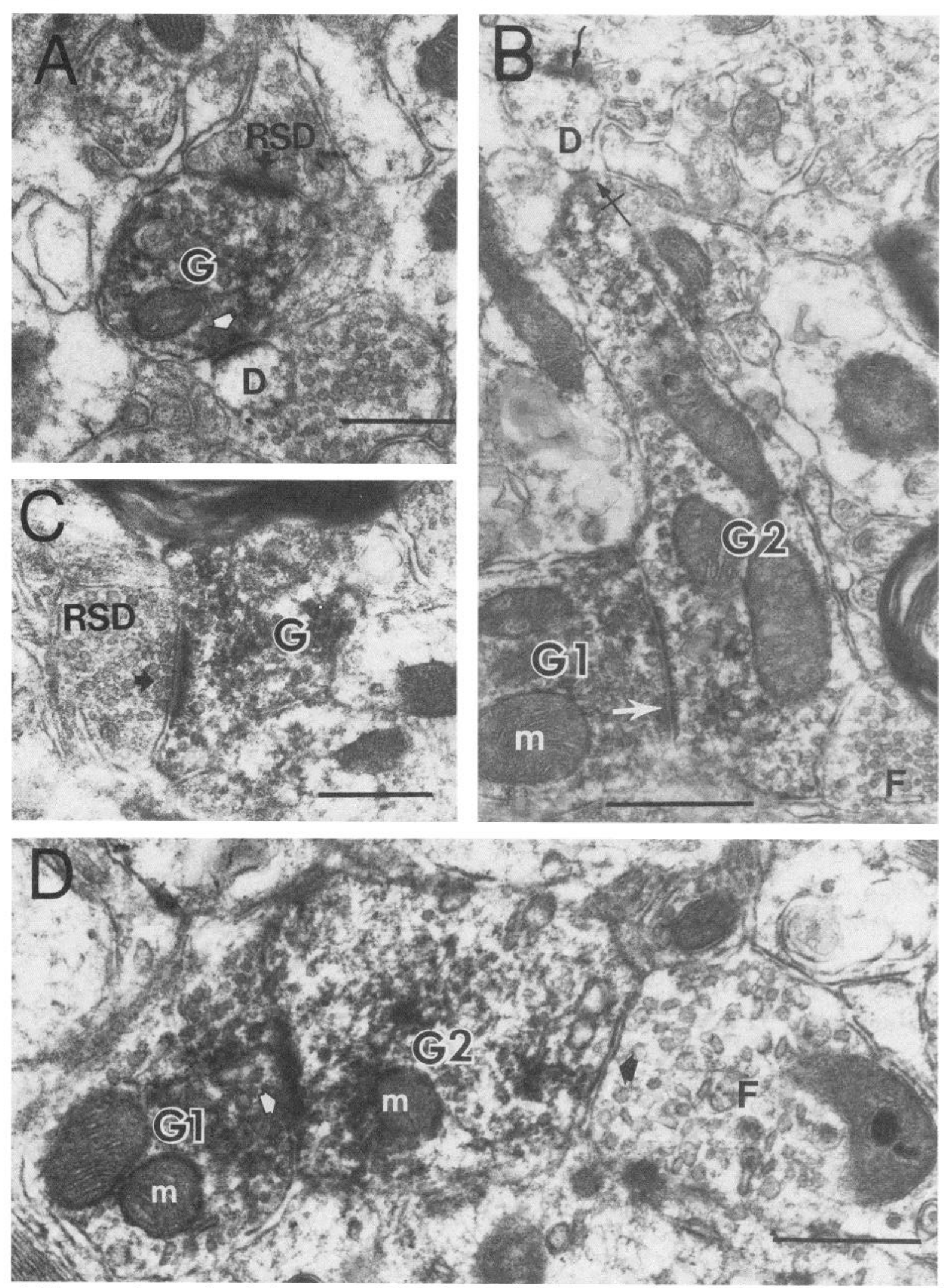

Figure 8. Electron micrographs of other types of GAD+ synaptic relationships found in the dLGN. A, A small diameter unlabeled terminal $(R S D)$ contains round vesicles and makes an asymmetric contact (black arrow) onto a GAD+ profile $(G)$ that, in turn, makes an asymmetric synaptic contact (white arrow) with a small unlabeled dendritic spine $(D)$, thereby completing a serial synapse. B, A clearly symmetric synaptic contact (white arrow) lies between two GAD+ profiles ( $G 1$ and $G 2$ ). G1 contains many synaptic vesicles, whereas G2 lacks synaptic vesicles near the contact. G2 contains a few vesicles at what may be a synaptic contact (crossed arrow) onto a small dendrite $(D)$, suggesting that $\mathrm{G} 2$ is a presynaptic dendrite. $\mathrm{D}$ also receives another synaptic contact from an adjacent unlabeled process (bent arrow). G2 is adjacent to an unlabeled process filled with flattened vesicles $(F)$. 
to an unlabeled F profile containing clearly flattened vesicles. Although GAD labeling in this longitudinal process is sparse, it can be observed at higher magnification on the surfaces of synaptic vesicles and in the cytoplasm (Fig. 9, $B$ and $C$, asterisks). Another frequent synaptic configuration consists of single $\mathrm{GAD}+$ profile which is presynaptic to a small unlabeled dendrite. This is especially common in the interlaminar zones (Fig. 12) but can also be found within the laminae. In these cases, the GAD+ profile is filled with vesicles and has numerous mitochondria. This type of contact is not related to the synaptic triads involving the retinal axon terminals.

The most common type of unlabeled terminal that is presynaptic to GAD+ synaptic profiles appears to be from retinal ganglion cell axons (Figs. 7 to 9 and 11). These can be identified on the basis of morphological criteria that are well established for the monkey dLGN (Guillery, 1971; Wilson and Hendrickson, 1981). Retinal terminals contain round synaptic vesicles, are large in diameter, and their numerous mitochondria have a pale appearance, hence the term RLP adopted by Guillery (1971). They may also contain neurofilaments, form multiple nonsynaptic adhesions with dendrites, and establish asymmetric synaptic specializations. In RLP terminals, triangular-shaped presynaptic dense projections are sometimes present (Figs. $7 B$ and $11 B$ ). In most instances when RLP terminals contact GAD+ profiles, the synaptic vesicles and associated GAD+ reaction product are sufficiently removed from the region of postsynaptic density so that the typical asymmetry of these junctions is easily recognized (Figs. $9, A$ and $B$ and $11, A$ and $B$ ). RLP terminals are not often found in presynaptic apposition to GAD+ structures that do not contain synaptic vesicles (but see Fig. $6 A$ ).

Unlabeled terminal types other than RLP terminals are much less commonly found in synaptic contact with GAD+ profiles. However, one of these resembles the RSD terminals of Guillery (1971), some of which are cortical in origin (Wong, 1970). These contain round vesicles, perhaps one dark mitochondrion, are small in diameter, and make a prominent asymmetric contact (Fig. 8, $A$ and $C$ ). RSD terminals are found presynaptic to GAD+ profiles in both laminar and interlaminar zones. Another unlabeled terminal type which also is presynaptic to GAD+ profiles is a pale $\mathrm{F}$ process that contains many scattered flat vesicles and makes a symmetric contact (Figs. 8, $B$ and $D$ and $9, C$ and $D$ ). This type may represent either another population of $F$ type synaptic profiles that is distinct from the GAD+ synaptic profiles or is unlabeled due to a false negative artifact (see Vaughn et al., 1981 for discussion).

\section{Discussion}

This investigation of GAD immunocytochemical staining in the monkey geniculate complex has resulted in four main observations: (1) there is a small but consistent population of GAD+ neurons within all layers of the nucleus; (2) the dLGN neuropil contains many GAD+ synaptic profiles of the $\mathrm{F}$ type that are more heavily concentrated in the laminar zones and are closely related to the retinal input; (3) some GAD+ profiles originate from presynaptic dendrites; and (4) the RNT also contains many GAD+ neurons whereas the neighboring vLGN exhibits only GAD+ neuropil with no detectable perikaryal labeling.

\section{$G A D+$ reactivity in the $d L G N$}

$G A D+$ neurons. The question of whether or not the monkey dLGN contains interneurons which have processes and connections that are confined to the nucleus has remained unresolved for many years, although the indirect evidence for their presence in the dLGN is extensive, as reviewed in the introduction. Taken together, these various experimental approaches strongly suggest that the monkey dLGN contains a small population of interneurons, some of which have presynaptic dendrites. Our immunocytochemical results suggest that at least some of these interneurons in the dLGN are GAD +. These small scattered GAD+ neurons have a large nucleus with an infolded nuclear membrane and thick, relatively straight dendrites. At the EM level, the dLGN neuropil contains GAD+ dendrites that are both pre- and postsynaptic and that are similar in morphology

Figure 8 continued.

$C$, An unlabeled terminal $(R S D)$ makes a prominent asymmetric contact onto a GAD+ profile $(G)$. This was taken from an interlaminar zone so that the unlabeled terminal is not retinal in origin. $D$, A GAD+ profile (G1) is presynaptic to another GAD+ profile (G2) at the white arrow. Both contain ill-defined cytoplasmic label, and mitochondria $(m)$, cisternae, and synaptic vesicles coated with GAD+ reaction product. The synaptic vesicles in G1 are oval in form, more numerous, and abut the synaptic specialization, whereas organelles and associated label largely avoid the immediately subjacent postsynaptic region in G2. Synaptic vesicles are sparse in G2. An unlabeled profile $(F)$ contains distinctly flattened vesicles and is in close contact with G2 (black arrow), although a synaptic contact is not clearly visible. Scale bars $=0.5 \mu \mathrm{m}$.

Figure 9. Electron micrographs of a longitudinally sectioned, lightly labeled GAD+ presynaptic dendrite $(G+)$ that exhibits several types of synaptic contacts. $A$, This low magnification view shows a varicose presymaptic dendrite that is both pre- and postsynaptic. It contains sparse GAD+ reaction product (asterisks) that is mainly associated with synaptic vesicles. Scale bar $=0.5 \mu \mathrm{m}$. $B$, The large GAD+ varicosity takes part in a synaptic complex where it is postsynaptic to an RLP terminal (upper black arrow). Synaptic vesicles in the G+ profile avoid this region, but they accumulate where it makes a symmetric synaptic contact (white arrow) onto a proximal dendrite $(D)$ that is also postsynaptic (lower black arrow) to the RLP terminal, completing a synaptic triad. Scale bar $=0.05 \mu \mathrm{m}$. $C$, The small GAD+ varicosity is postsynaptic to an unlabeled, pale profile containing distinctly flattened vesicles $(F)$ that cluster near an asymmetric synaptic specialization (open arrow). An accumulation of GAD+ pleomorphic vesicles (asterisk) lies in the varicosity at a distance from the postsynaptic region. Same magnification as Figure $8 B$. $D$, Another example of an unlabeled $F$ profile containing many flat vesicles $(F)$ that is presynaptic to a GAD-labeled synaptic profile $(G)$. Note the asymmetric contact beneath the arrowhead. Same magnification as Figure $8 B$. 



Figure 9 

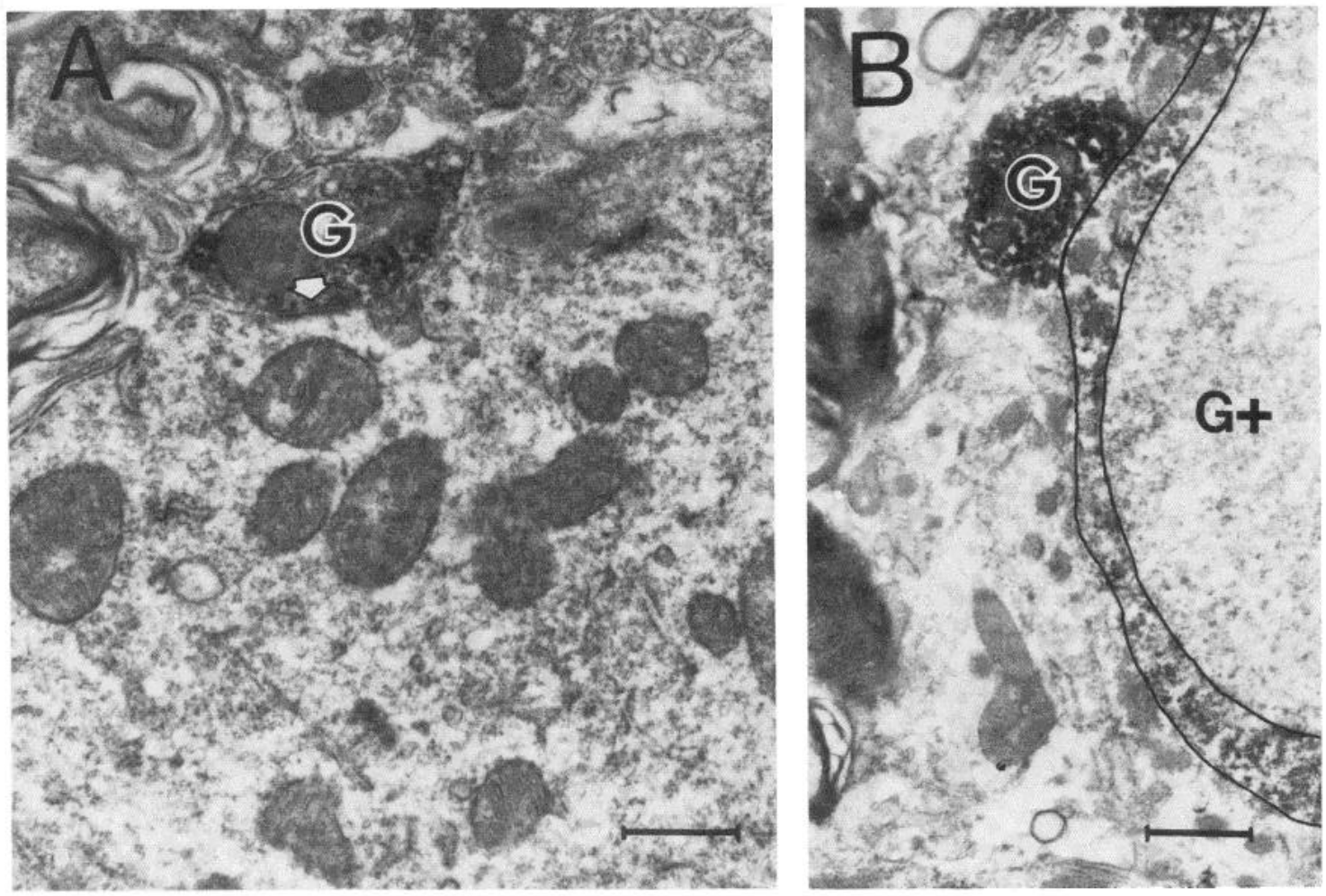

Figure 10. Electron micrographs of GAD+ synaptic contacts onto dLGN somata. $A$, A GAD-labeled profile $(G)$ synapses onto an unlabeled neuronal cell body at a symmetric synaptic specialization (white arrow). $B, \mathrm{~A}$ labeled profile $(G)$ contacts a labeled cell body $(G+)$, although poor ultrastructural preservation does not permit visualization of a synaptic specialization. The cell and nuclear membranes have been outlined for clarity. Note the relatively small amount of perikaryal cytoplasm in this labeled neuron compared to the unlabeled neuron in $A$. Scale bars $=0.5 \mu \mathrm{m}$.

and synaptic relationships to presynaptic dendrites shown by other authors (LeVay, 1971; Pasik et al., 1973; Hamori et al., 1974; Winfield, 1980). In addition, these cells are similar to $\left[{ }^{3} \mathrm{H}\right] \mathrm{GABA}$-accumulating neurons in cat dLGN (Sterling and Davis, 1980) that have been suggested to be interneurons on the basis of similar criteria. Moreover, our findings in monkey are very similar to a recent study of GAD localization in rat dLGN (Ohara et al., 1983). The rat dLGN contains a population of GAD+ neurons, some of which have presynaptic dendrites, which closely resembles the previously characterized interneurons from Golgi impregnations (Lieberman and Webster, 1972; Lieberman, 1973). In rat dLGN, GAD+ synaptic profiles, including presynaptic dendrites, are commonly encountered in the neuropil and have the same distribution and similar morphology as we have found for monkey dLGN neuropil. However persuasive this indirect evidence may be, the critical experiment still remains to be done, namely to demonstrate by double labeling techniques that these GAD+ cells do not project to visual cortex. This is especially important in view of the intracellular filling experiments of Friedlander et al. (1981), who find that cat dLGN contains some neurons that resemble the postulated interneurons in having appendage-laden dendrites, but their axon first ramifies locally and then projects to visual cortex.

GAD+ neuropil. At both the LM and EM level, it is clear that the dLGN neuropil is rich in GAD+ processes and synaptic profiles. Because of the altered ultrastructure resulting from GAD staining, however, it has been difficult to classify GAD+ synaptic profiles according to the traditional EM criteria of vesicle shape and symmetry of the synaptic specialization. It should be emphasized, though, that the regularity with which GAD+ profiles are found as both pre- and postsynaptic elements indicates that some are presynaptic dendrites, and as such would fall into the F category as described in conventional electron microscopy. The same difficulties apply to the individual GAD+ synaptic profiles that occur outside of synaptic complexes. When compared to surrounding unlabeled neuropil elements, these profiles appear to be of the $\mathrm{F}$ type, even though their postsynaptic densities are not strictly symmetric. This identification is strengthened by previous reports in rat of individual $\mathrm{F}$ terminals arising from the RNT (Ohara et al., 1980; Montero and Scott, 1981), the neurons of which are

Figure 11. Electron micrographs of GAD+ profiles $(G)$ that are postsynaptic to unlabeled, retinal terminals $(R L P)$ that contain round vesicles and several pale mitochondria $(m)$. $A$, This RLP terminal contains a characteristic neurofilamentous core and makes an asymmetric synaptic contact (arrow) with the GAD+ profile. $B$, This RLP terminal makes an asymmetric contact (arrow) that also shows presynaptic dense triangular projections. Both GAD+ profiles contain mitochondria $(m)$ whose surfaces are associated with label, and a pleomorphic population of synaptic vesicles that are outlined by intensely labeled surfaces. Scale bars $=0.5 \mu \mathrm{m}$. 

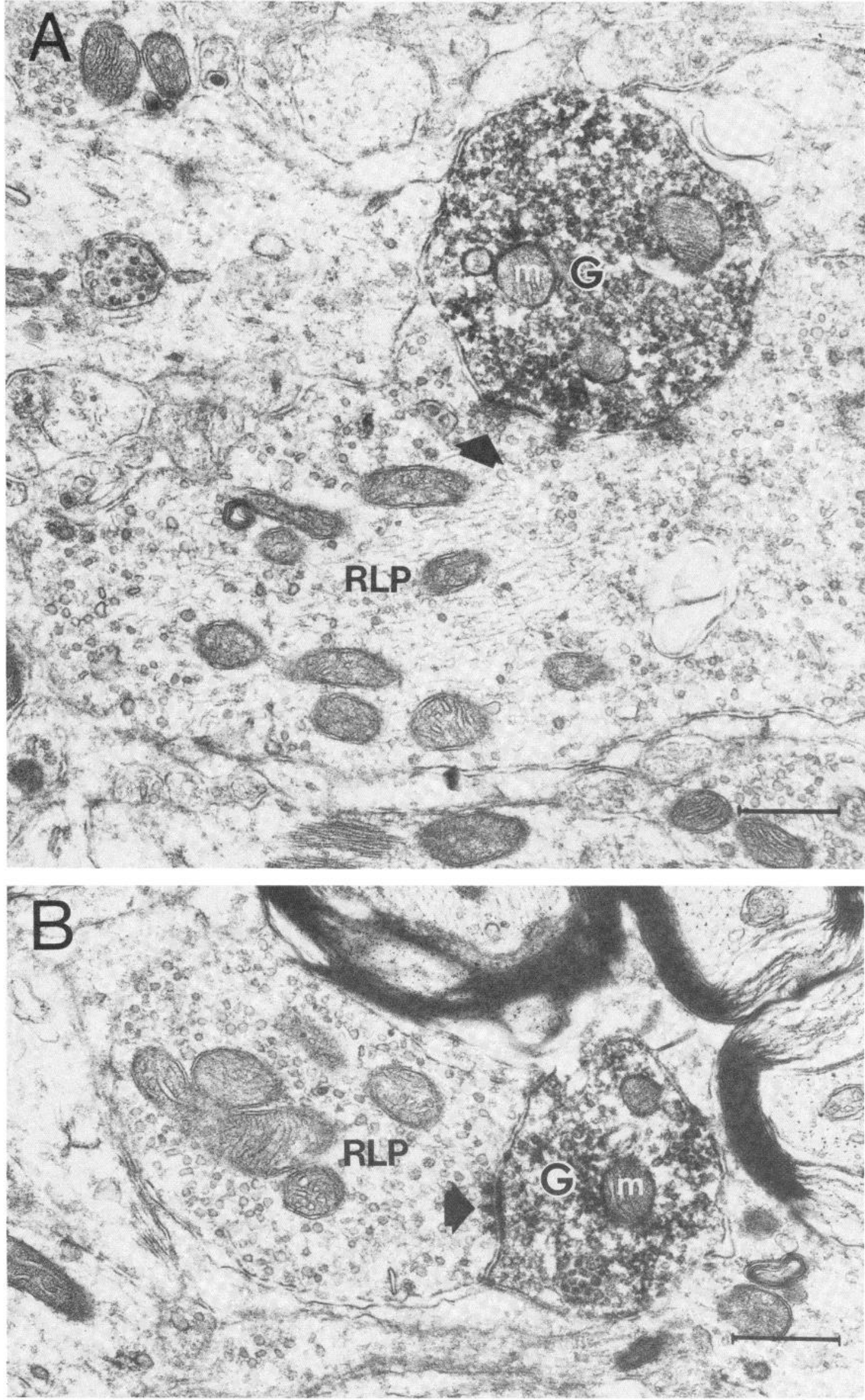

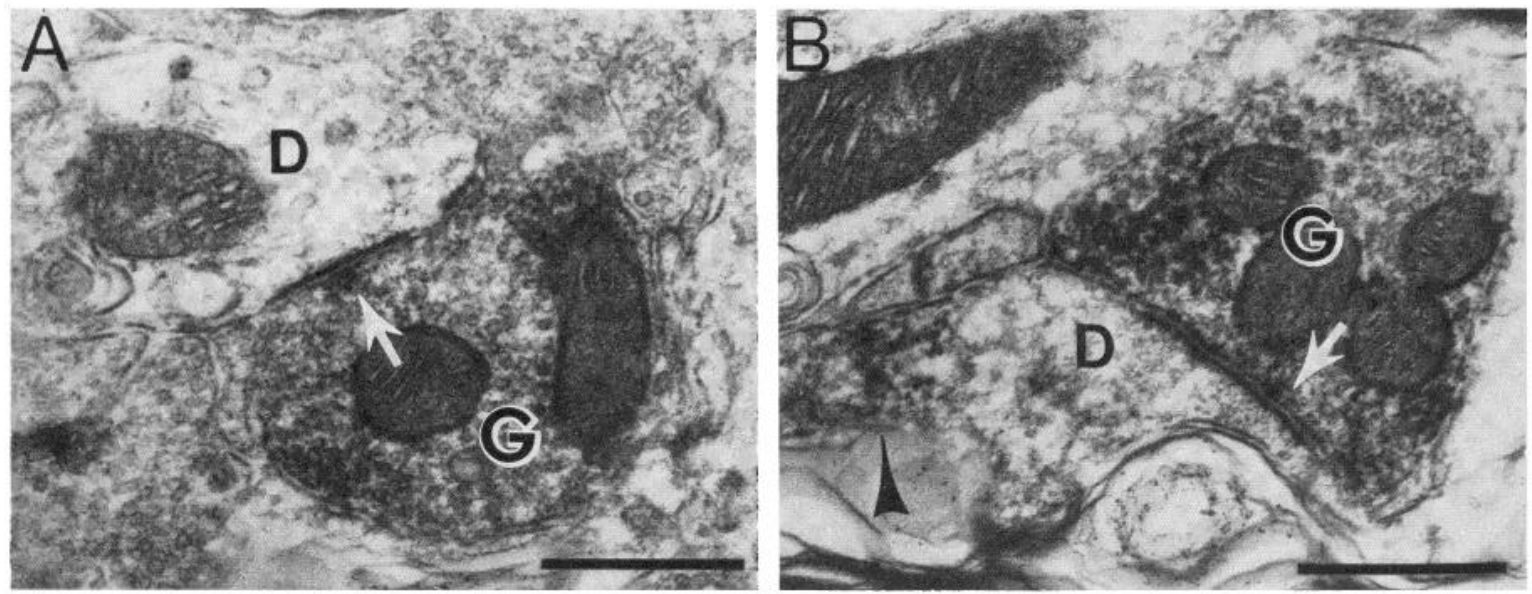

Figure 12. Electron micrographs of GAD+ presynaptic profiles $(G)$ from the interlaminar zones. In $A$, the dendrite $(D)$ is unlabeled, but in $B$ there may be diffuse GAD+ material in the dendrite $(D)$ at the arrowhead. Both GAD+ profiles are filled with synaptic vesicles, contain several mitochondria, and make contacts (white arrows) which are intermediate in postsynaptic density. Scale bars $=0.5 \mu \mathrm{m}$.

known to be GABAergic (Houser et al., 1980). Therefore, when the GAD+ synaptic profiles identified in this study are considered against a background of the numerous studies of geniculate circuitry using conventional EM, and by comparison with the unlabeled terminals in the GAD processed material, they would fit into the $\mathrm{F}$ classification.

Several points need to be emphasized about the spatial distribution of GAD+ synaptic profiles. First, although they are found throughout the dLGN, they are more concentrated in the six geniculate laminae as well as in the $\mathrm{S}$ layers and the intercalcated zones, closely paralleling the retinal input. This relationship is further borne out at the EM level, where GAD+ synaptic profiles are especially numerous in the synaptic glomeruli which contain retinal terminals. Specifically, the intervening $\mathrm{F}$ processes in synaptic triads are frequently GAD+ profiles that are simultaneously postsynaptic to unlabeled retinal terminals and presynaptic to unlabeled dendrites that are also postsynaptic to the same retinal terminals. This morphological arrangement places these profiles in a strategic position to play a major role in GABA-mediated, retinally driven, feed-forward inhibition (Stevens and Gerstein, 1976; Dubin and Cleland, 1977). Because at least some of the GAD+ profiles appear to originate from presynaptic dendrites, this also implicates the GAD + dLGN interneurons in this process.

Second, the magnocellular layers are the most intensely stained portion of the dLGN. This is in good agreement with previous EM reports showing that magnocellular layers contain more $\mathrm{F}$ profiles than the parvocellular layers (Winfield, 1980; Wilson and Hendrickson, 1981). In addition, Rodieck and Dreher (1979) have found suppression of electrophysiological activity by the nondominant eye in the magno- but not parvocellular layers of monkey dLGN, suggesting a greater inhibitory activity that may correlate with this heavier GAD labeling in the magnocellular layers.

Third, we find that individual GAD + synaptic profiles make contact onto neuronal somata and distal and prox- imal dendrites in both the laminar and interlaminar zones. This suggests that GABA has a widespread influence on the properties of dLGN neurons in addition to its relationship to retinal input in the glomeruli. This would be especially true of the individual GAD+ profiles in the interlaminar zones where retinal input is lacking (reviewed by Wilson and Hendrickson, 1981). Moreover, GABA pathways may directly interact as GAD+ profiles synapse onto GAD+ somata and onto other GAD+ processes that resemble presynaptic dendrites.

It should be noted that an unlabeled, characteristically pale, $\mathrm{F}$ type profile is sometimes found in a presynaptic relationship to GAD+ profiles. Sterling and Davis (1980) also report a similar type of $\mathrm{F}$ profile that does not show $\left[{ }^{3} \mathrm{H}\right] \mathrm{GABA}$ uptake in cat dLGN. There are technical reasons why these $\mathrm{F}$ profiles could be GABAergic but do not stain for GAD (Vaughn et al., 1981), but their presence in both cat and monkey dLGN also raises the possibility that GABA may not be the exclusive transmitter for the F profile type.

Origins for GAD+ pathways in $d L G N$. Within the immediate vicinity of the dLGN, we find only one other population of GAD+ neurons, those of the RNT. This confirms a previous report by Houser et al. (1980), who showed that virtually all of the neurons in the rat RNT are GAD+. There are a number of recent reports that emphasize a pathway from RNT to the dLGN (Jones, 1975; Burke and Cole, 1978; Ohara et al., 1980; Montero and Scott, 1981; Hale et al., 1982). Labeling studies using terminal degeneration (Ohara et al., 1980) or $\left[{ }^{3} \mathrm{H}\right]$ proline (Montero and Scott, 1981) show that the RNT axon terminals in rat dLGN are of the F type. RNT terminals are not found in synaptic triads; rather, they are distributed onto the neuronal somata and proximal and distal dendrites in simple presynaptic relationships similar to some of the individual GAD+ profiles observed in this study. Given that the monkey RNT is almost entirely composed of GAD+ neurons and that its cells project to dLGN (Jones, 1975; A. E. Hendrickson, unpublished results), and assuming that the RNT axons have the 
same distribution in monkey and rat, it is likely that many of the individual GAD+ profiles ending on somata and small dendrites originate in the RNT.

The vLGN is an unlikely source of GAD+ profiles in the monkey, as this nucleus contains no observable GAD+ somata. Scattered GAD+ neurons are found lying outside the dLGN capsule but these would seem to be too sparse to provide a significant input. Therefore, we suggest that the presynaptic dendrites of the GAD+ dLGN interneuron are the most likely source for the GAD+ synaptic profiles which are associated with retinal axons in glomeruli. Individual GAD+ synaptic profiles outside glomeruli are likely to be axons of RNT origin. The specific proportion and distribution of RNT versus intrinsic input and the possibility that a long axon pathway to dLGN is GABAergic remain to be determined in future studies.

\section{References}

Barber, R. P., and K. Saito (1976) Light microscopic visualization of GAD and GABA-T in immunocytochemical preparations of rodent CNS. In GABA in Nervous System Function, E. Roberts, T. N. Chase, and D. B. Tower, eds., pp. 113-132, Raven Press, New York.

Burke, W., and A. M. Cole (1978) Extraretinal influences on the lateral geniculate nucleus. Rev. Physiol. Biochem. Pharmacol. 80: 105-166.

Campos-Ortega, J. A., P. Glees, and V. Neuhoff (1968) Ultrastructural analysis of individual layers in the lateral geniculate body of the monkey. Z. Zellforsch. 87: 82-100.

Curtis, D. R., and A. K. Tebecis (1972) Bicuculline and thalamic inhibition. Exp. Brain Res. 16: 210-218.

Dineen, J. T., A. Hendrickson, and E. G. Keating (1982) Alterations of retinal inputs following striate cortex removal in adult monkey. Exp. Brain Res. 47: 446-456.

Dubin, M. E., and B. G. Cleland (1977) Organization of visual inputs to interneurons of lateral geniculate nucleus of the cat. J. Neurophysiol. 40: 410-427.

Famiglietti, E. V., Jr., and A. Peters (1972) The synaptic glomerulus and the intrinsic neuron in the dorsal lateral geniculate nucleus of the cat. J. Comp. Neurol. 144: 285-334.

Friedlander, M. J., C. -S. Lin, L. R. Stanford, and S. M. Sherman (1981) Morphology of functionally identified neurons in lateral geniculate nucleus of the cat. J. Neurophysiol. 46: 80-129.

Guillery, R. W. (1971) Patterns of synaptic interconnections in the dorsal lateral geniculate nucleus of cat and monkey: A brief review. Vision Res. Suppl. 3: 211-228.

Guillery, R. W., and M. Colonnier (1970) Synaptic patterns in the dorsal lateral geniculate nucleus of the monkey. Z. Zellforsch. 103: 90-108.

Hale, P. T., A. V. Sefton, L. A. Baur, and L. J. Cottee (1982) Interrelations of the rat's thalamic reticular and dorsal lateral geniculate nuclei. Exp. Brain Res. 45: 217-229.

Hamori, J., T. Pasik, P. Pasik, and J. Szentagothai (1974) Triadic synaptic arrangements and their possible significance in the lateral geniculate nucleus of the monkey. Brain Res. 80: 379-393.

Hendrickson, A., M. E. Wilson, and M. J. Toyne (1970) The distribution of optic nerve fibers in Macaca mulatta. Brain Res. 23: 425-427.

Hendrickson, A. E., S. P. Hunt, and J. -Y. Wu (1981) Immunocytochemical localization of glutamic acid decarboxylase in monkey striate cortex. Nature 292: 605-607.

Houser, C. R., J. E. Vaughn, R. P. Barber, and E. Roberts
(1980) GABA neurons are the major cell type of the nucleus reticularis thalami. Brain Res. 200: 341-354.

Jones, E. G. (1975) Some aspects of the organization of the thalamic reticular complex. J. Comp. Neurol. 162: 285-308.

LeVay, S. (1971) On the neurons and synapses of the lateral geniculate nucleus of the monkey and the effects of eye enucleation. Z. Zellforsch. 113: 396-419.

LeVay, S., and D. Ferster (1979) Proportion of interneurons in the cat's lateral geniculate nucleus. Brain Res. 164: 304-308.

Lieberman, A. R. (1973) Neurons with presynaptic perikarya and presynaptic dendrites in the rat lateral geniculate nucleus. Brain Res. 59: 35-59.

Lieberman, A. R., and K. E. Webster (1972) Aspects of the synaptic organization of intrinsic neurons in the dorsal lateral geniculate nucleus. An ultrastructural study of the normal and the experimentally deafferented nucleus in the rat. J. Neurocytol. 3: 677-710.

MacLaughlin, B. J., J. G. Wood, K. Saito, R. Barber, J. E. Vaughn, E. Roberts, and J. -Y. Wu (1974) The fine structural localization of glutamate decarboxylase in synaptic terminals of rodent cerebellum. Brain Res. 76: 377-391.

Montero, J. M., and G. L. Scott (1981) Synaptic terminals in the dorsal lateral geniculate nucleus from neurons of the thalamic reticular nucleus: A light and clectron microscopic study. Neuroscience 6: 2561-2577.

Norden, J. J., and J. H. Kaas (1978) The identification of relay neurons in the dorsal lateral geniculate nucleus of monkeys using horseradish peroxidase. J. Comp. Neurol. 182: 707-726.

Ogren, M., and A. Hendrickson (1976) Pathways between striate cortex and subcortical regions in Macaca mulatta and Saimiri sciureus: Evidence for a reciprocal pulvinar connection. Exp. Neurol. 53: 780-800.

Ogren, M. P., and A. E. Hendrickson (1979) The structural organization of the inferior and lateral subdivisions of the Macaca monkey pulvinar. J. Comp. Neurol. 188: 147-178.

Ogren, M. P., A. E. Hendrickson, J. Vaughn, R. P. Barber, and J. - Y. Wu (1982) GABAergic neurons and synapses in monkey dorsal lateral geniculate: A light and electron microscopic immunohistochemical analysis. Soc. Neurosci. Abstr. 8: 262.

Ohara, P. T., A. J. Sefton, and A. R. Lieberman (1980) Mode of termination of afferents from the thalamic reticular nucleus of the rat. Brain Res. 197: 503-506.

Ohara, P. T., A. R. Lieberman, S. P. Hunt, and J. -Y. Wu (1983) Neural elements containing glutamic acid decarboxylase (GAD) in the dorsal lateral geniculate nucleus of the rat; immunohistochemical studies by light and electron microscopy. Neuroscience, in press.

Partlow, G. D., M. Colonnier, and J. Szabo (1977) Thalamic projections of the superior colliculus in the rhesus monkey, Macaca mulatta: A light and electron microscopic study. J. Comp. Neurol. 171: 285-318.

Pasik, P., T. Pasik, J. Hamori, and J. Szentagothai (1973) Golgitype interneurons in the neuronal circuit of the monkey lateral geniculate nucleus. Exp. Brain Res. 17: 18-34.

Polyak, S. (1957) The Vertebrate Visual System, pp. 354-376, University of Chicago Press, Chicago.

Ralston, H. J., III, and M. M. Herman (1969) The fine structure of neurons and synapses in the ventrobasal thalamus of the cat. Brain Res. 14: 77-97.

Ribak, C. E., J. E. Vaughn, and K. Saito (1978) Immunocytochemical localization of glutamic acid decarboxylase in neuronal somata following colchicine inhibition of axonal transport. Brain Res. 140: 315-332.

Ribak, C. E., J. E. Vaughn, and R. P. Barber (1981) Immunocytochemical localization of GABAergic neurons at the electron microscopic level. Histochem. J. 13: 555-582.

Rodieck, R. W., and B. Dreher (1979) Visual suppression from 
nondominant eye in the lateral geniculate nucleus. A comparison of cat and monkey. Exp. Brain Res. 35: 465-477.

Saini, K. D., and L. J. Garey (1981) Morphology of neurons in the lateral geniculate nucleus of the monkey. Exp. Brain Res. 42: $235-248$.

Saito, K., J. -Y. Wu, T. Matsuda, and E. Roberts (1974) Immunocytochemical comparisons of vertebrate glutamic acid decarboxylase. Brain Res. 65: 277-285.

Sterling, P., and T. L. Davis (1980) Neurons in cat lateral geniculate nucleus that concentrate exogenous $\left[{ }^{3} \mathrm{H}\right]-\gamma$-aminobutyric acid (GABA). J. Comp. Neurol. 192: 737-749.

Sternberger, L. A. (1979) Immunocytochemistry, pp. 104-169, John Wiley \& Sons, Inc., New York.

Stevens, J. K., and G. L. Gerstein (1976) Interactions between cat lateral geniculate neurons. J. Neurophysiol. 39: 239-256.

Sumitomo, I., M. Nakamura, and K. Iwama (1976) Location and function of the scattered interneurons of rat lateral geniculate body. Exp. Neurol. 51: 110-123.

Vaughn, J. E., R. F. Barber, C. E. Ribak, and C. R. Houser (1981) Methods for the immunocytochemical localization of proteins and peptides involved in neurotransmission. In Cur- rent Trends in Morphological Techniques, J. E. Johnson, Jr., ed., Vol. III, pp. 33-70, CRC Press, Boca Raton, FL.

Wilson, J. R., and A. E. Hendrickson (1981) Neuronal and synaptic structure of the dorsal lateral geniculate nucleus in normal and monocularly deprived Macaca monkeys. J. Comp. Neurol. 197: 517-539.

Winfield, D. A. (1980) The synaptic organization of glomeruli in the magnocellular and parvocellular laminae of the lateral geniculate nucleus in the monkey. Brain Res. 198: 55-62.

Wong, M. T. T. (1970) Somato-dendritic and dendro-dendritic synapses in the squirrel monkey lateral geniculate nucleus. Brain Res. 20: 135-139.

Wu, J. -Y., T. Matsuda, and E. Roberts (1973) Purification and characterization of glutamate decarboxylase from mouse brain. J. Biol. Chem. 248: 3029-3034.

Wu, J. -Y., C. -T. Lin, C. Brandon, T. -S. Chan, H. Mohler, and J. G. Richards (1982) Regulation and immunocytochemical characterization of glutamic acid decarboxylase. In Cytochemical Methods in Neuroanatomy, V. Chan-Palay and S. L. Palay, eds., pp. 279-296, Alan R. Liss, Inc., New York. 